\title{
Exercise Capacity of the Patients with Myocardial Infarction
}

\author{
Toshikatsu Sada, M.D., Kuan-Min Su, M.D., Naomi Amano, M.D. \\ NaOki Hayashi, M.D., Ineko Tawara, M.D. \\ and Masaya Takeuchi, M.D.
}

\begin{abstract}
This study was conducted to investigate the functional capacity in postmyocardial infarction. Eighty-four multistage treadmill exercise tests were performed on 60 patients, none of whom had had any formal rehabilitation or regular exercise. There were 50 men and 10 women, ranging in age from 30 to 81 with an average age of 60 . The time interval between the acute attack and the exercise test ranged from one month to 9 years.

Even though severe infarction affects the exercise capacity for a long time after an acute attack, its effect on cardiac function was more obvious than that on physical capacity.

Age was the most important determinant of physical capacity, and the slope of decreasing physical capacity with age in patients with infarct was the same as that in normal subjects. Cardiac function also decreased with age. However, during the early recovery phase, cardiac function was influenced by the severity of infarction and the influence of age could not be established.

There was no significant correlation between early ambulation and physical capacity. The beneficial effects of early ambulation may be lost if physical activity is discontinued for some time after the acute attack.

The physical capacity increased 2-3 years after the acute attack, but myocardial function did not change significantly.
\end{abstract}

$\mathbf{T}^{\mathrm{t}}$ HE approach to cardiac rehabilitation has changed during the last decade. It is now widely believed that prolonged bed rest and restricted activity bring about cardiovascular and physical deterioration, 1 and that regular exercise prevents the development and progression of ischemic heart disease. There are many studies indicating the beneficial effects of physical activity? However, there have been few reports ${ }^{3}$ on the natural history of cardiovascular and physical capacities of patients with myocardial infarction. It is important to study the recovery course after myocardial infarction in order to

Key Words:

Myocardial infarction

PRP

METs

Exercise test evaluate the effects of rehabilitation and exercise training. We studied the cardiac and the physical function in post-myocardial infarction.

\section{MATERIALS AND METHODS}

Subjects: Sixty patients documented to have had myocardial infarction (39 anterior and 21 inferior) underwent exercise testing. There were 50 men and 10 women, ranging in age from 30 to 81 with an average of 60 . None of these patients had participated in any formal rehabilitation or regular exercise program. The time interval between the acute infarction and the exercise testing ranged from one month to 9 years.

Exercise Testing: Eighty-four multistage exercise tests were performed using a modified Bruce protocol (Table I). Exercise was continued

The Fourth Department of Internal Medicine, Faculty of Medicine, The University of Tokyo and Showa Hospital, Tokyo, Japan

Mailing address: Toshikatsu Sada, M.D., The Fourth Department of Internal Medicine, Faculty of Medicine, The University of Tokyo, 3-28-6 Mejirodai, Bunkyo-ku, Tokyo 112, Japan 
TABLE I EXERCISE PROTOCOL

\begin{tabular}{ccrc}
\hline Stage & $\begin{array}{c}\text { Speed } \\
\text { (mile/hour) }\end{array}$ & \multicolumn{2}{c}{$\begin{array}{c}\text { Grade } \\
\text { (\%) }\end{array}$} \\
\hline$I$ & 1.0 & 5 & Bruce's \\
$I I$ & 1.0 & 10 & Protocol \\
$I I I$ & 1.7 & 10 & $(I)$ \\
$I V$ & 2.5 & 12 & $(I I)$ \\
$V$ & 3.4 & 14 & $(I I I)$ \\
$V I$ & 4.2 & 16 & $(I V)$ \\
\hline
\end{tabular}

until the heart rate reached $85 \%$ of the agepredicted maximum value or one of the following signs or symptoms appeared: anginal pain, dyspnea, severe fatigue, serious arrhythmias, a horizontal or downsloping ST depression of 2 $\mathrm{mm}$ or a fall in systolic blood pressure. Bipolar lead electrocardiograms, $\mathrm{CC}_{2}, \mathrm{CC}_{5}$ and $\mathrm{ML}$ (manubrium to lumber region), were recorded at rest and during exercise. Blood pressure was measured using a cuff-sphygmomanometer at one-minute intervals throughout the procedure. The volume and content of oxygen and carbon dioxide components of the expired gas were measured continuously using an Expired Gas Analyzer IHO2-4 (San-ei Instruments, Tokyo). The oxygen consumption was calculated every minute. Oxygen consumption could not be measured in 8 cases during the exercise test.

The unpaired $t$-test and chi-square test were used for statistical analysis.

\section{RESULTS}

Forty-seven out of 84 exercise tests $(56.0 \%)$ were discontinued when the patient's heart rate reached the $85 \%$ limit of the age-predicted maximum heart rate. In the remaining 37 tests exercise was discontinued for the following reasons: subjective symptoms in $11(13.1 \%)$, an ST depression in $25(29.8 \%)$ and a fall in systolic blood pressure in one (1.2\%). There were no complications.

It is very difficult to express the severity of myocardial infarction quantitatively. In an attempt to estimate it, we used the peak value of serum creatine phosphokinase (CPK) and Peel's prognostic index 4 in the acute stage of the attack. The pressure rate product (PRP), which is considered to reflect myocardial oxygen consumption, 5,6 showed a negative correlation with CPK $(r=-0.31, p<0.01)$ and with Peel's
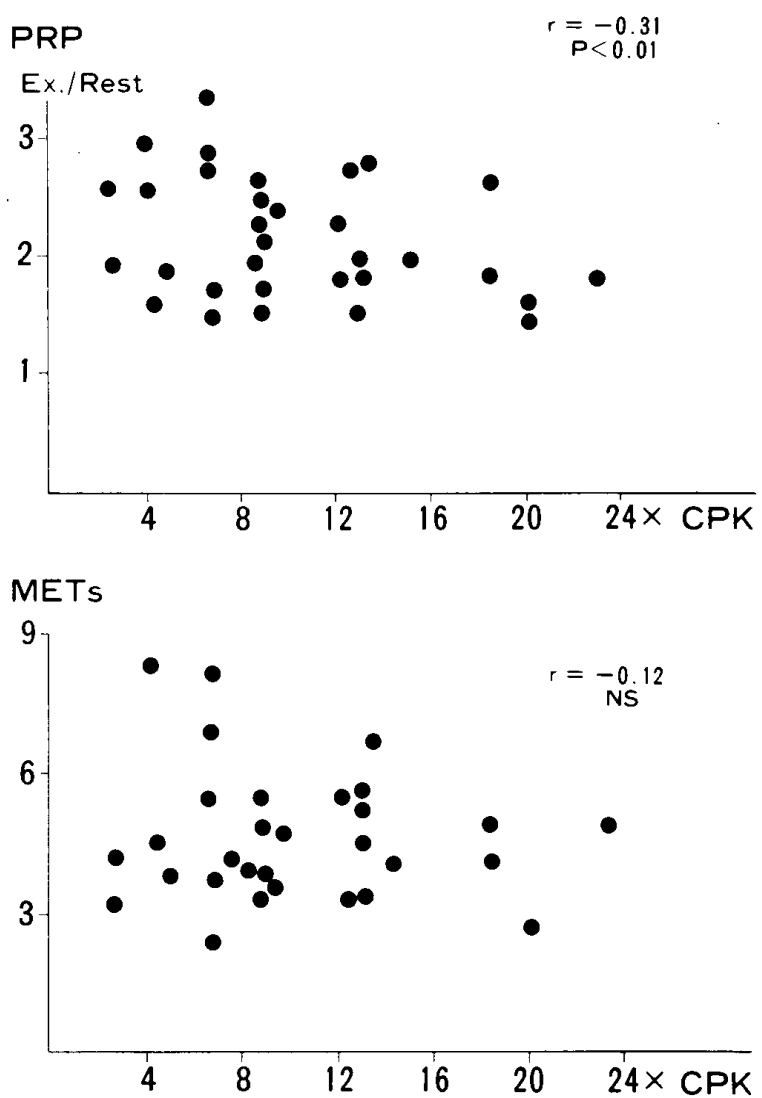

Fig. 1. Relationships between CPK and the PRP (upper panel), and the METs (lower panel). CPK: peak value of serum creatine phosphokinase expressed as a ratio to normal value. PRP: pressure rate product expressed as a ratio at the peak of exercise and at rest. PRP is correlated with CPK $(\mathrm{r}=-0.31, \mathrm{p}<0.01)$. Since CPK was not available in some cases, the total number was less than 84 .

TABLE II CORRELATIONS OF PRP AND METS WITH SEVERAL VARIABLES

\begin{tabular}{lcc}
\hline & PRP & METs \\
\hline CPK & -0.31 & $n s$ \\
Peel's prognostic index & -0.35 & -0.37 \\
Age & & \\
All cases & -0.41 & -0.44 \\
Within 6 months & $n s$ & -0.45 \\
After 6 months & -0.66 & -0.58 \\
Symptom-limited & & $\cdot$ \\
max. exercise & -0.36 & -0.59 \\
Within 6 month & $n s$ & -0.53 \\
After 6 month & -0.67 & -0.71 \\
\hline
\end{tabular}

$P R P=$ pressure rate product, ns $=$ not significant 

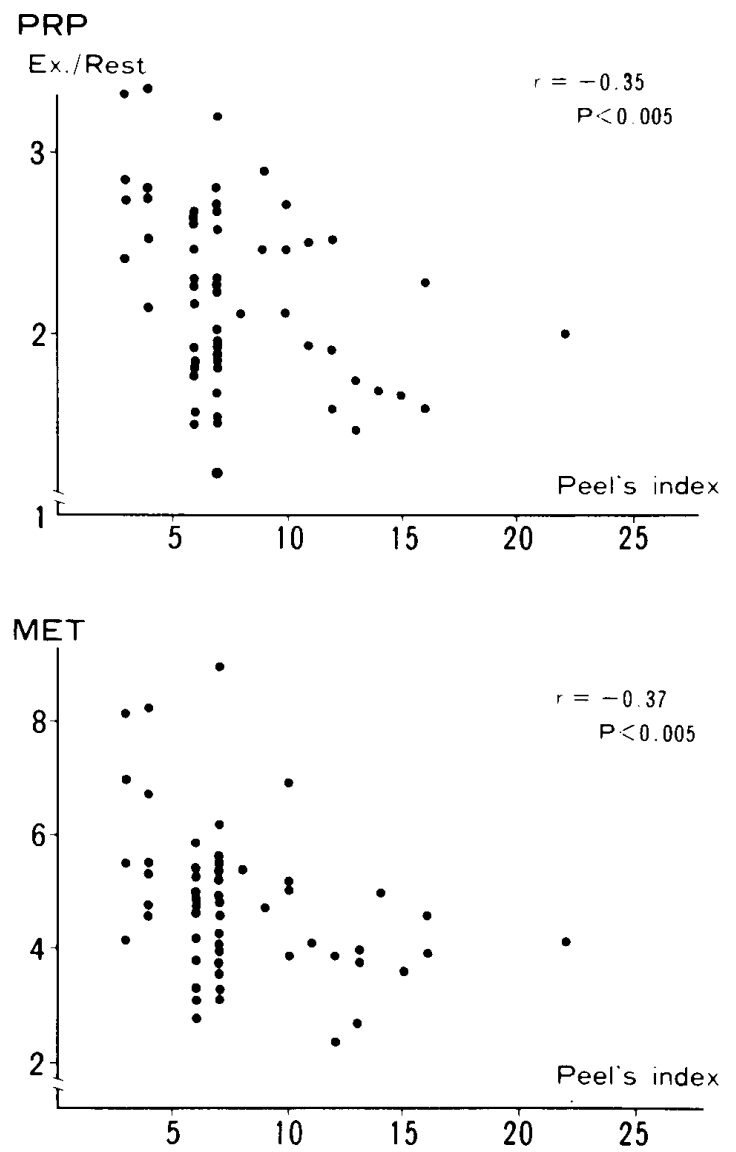

Fig.2. Relationships between Peel's prognostic index and the PRP (upper panel), and the METs (lower panel). There are significant correlations between Peel's index and the PRP $(r=-0.35, p<0.005)$, and the METs $(r=-0.37, p<0.005)$.

index $(r=-0.35, p<0.005)$, but the METs had a negative correlation only with Peel's index $(\mathrm{r}=-0.37, \mathrm{p}<0.005)$ (Figs. 1,2 and Table II). A severe myocardial infarction affected cardiac and physical capacity for a long period after the acute attack.

Age at the time of the exercise test was significantly correlated with the PRP $(r=-0.41, \mathrm{p}<$ $0.001)$ and with the METs $(r=-0.44, p<$ 0.001). Excluding those patients who stopped the exercise test when their heart rate reached $85 \%$ of their age-predicted maximum value, there were also significant correlations between the age and the PRP ( $\mathrm{r}=-0.36, \mathrm{p}<0.025)$, and age and the METs $(r=-0.59, \mathrm{p}<0.001)$ (Fig. 3). The PRP had no correlation with age within 6 months after the acute attack, but the METs had a negative correlation with age both within 6 months of the acute attack and afterwards (Table II).

There were no significant correlations between the PRP, the METs and the duration of
PRP
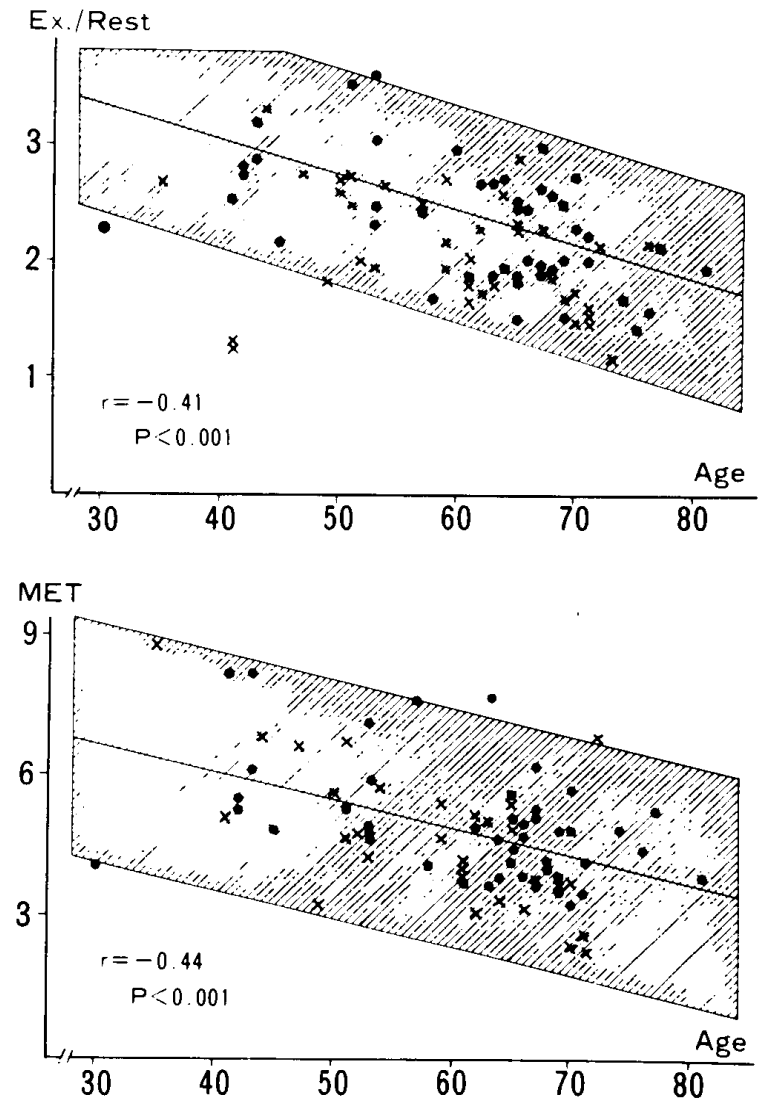

Fig. 3. Relationships between age and the PRP (upper panel), and the METs (lower panel). There are significant correlations between age and the PRP $(r=-0.41, p<0.001)$, and the METs $(r=-0.44, p<0.001)$. $x$ : the cases who stopped exercise due to reaching their target heart rate. Slope of the relationship between age and the METs is -0.1 , being the same as that in normal subjects.

ambulation. However, among the cases within 6 months after the attack, the patients who had a 2-week or longer ambulation achieved the exercise level of 5 METs or less during the test (Fig. 4).

Figure 5 shows the time course of the PRP and the METs after the acute myocardial infarction. The PRP was distributed over a wide range without any special pattern. On the other hand, oxygen consumption was less than 5 METs in many cases during the early phase of the recovery, increased duirng the second and the third year and decreased again thereafter.

\section{DISCUSSION}

The capacity for exercise in human beings can be estimated by measuring oxygen consumption? 

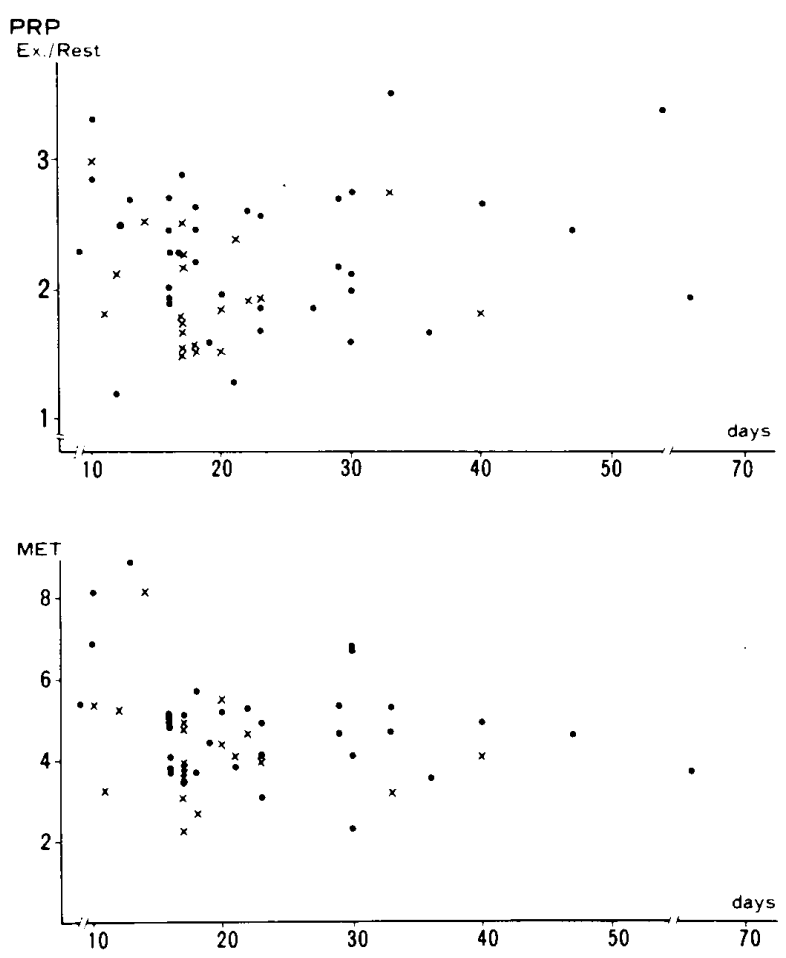

Fig.4. Relationship between the duration of ambulation and the PRP (upper panel), and the METs (lower panel). There are no significant correlations. $x$ : the cases within 6 months after the attack.

However, the myocardial and the total body oxygen consumption must be considered separately, because physical capacity is affected by both cardiac and peripheral factors. Total body oxygen consumption can be easily measured and expressed by the METs as we have done. The direct measurement of myocardial oxygen consumption is difficult, but the PRP is closely correlated with oxygen consumption in both normal subjects 5 and those with ischemic heart disease. 6 Thus, the METs and the PRP are used as indexes of the physical and the cardiac capacity of patients.

There were significant correlations between the PRP, CPK and Peel's prognostic index. Grande and Pedersen ${ }^{8}$ have demonstrated that the infarct size estimated from the CPK level correlates with the cardiac function soon after an attack of myocardial infarction. In the present study more than one half of the patients underwent the exercise test 2 or more years after the acute attack. Therefore, the severity of the infarction influences the myocardial function not only during the early recovery period, but for a long time afterwards. On the other hand, the METs had no correlation with CPK. Carter and Amundsen ${ }^{9}$ have reported that the METs and the
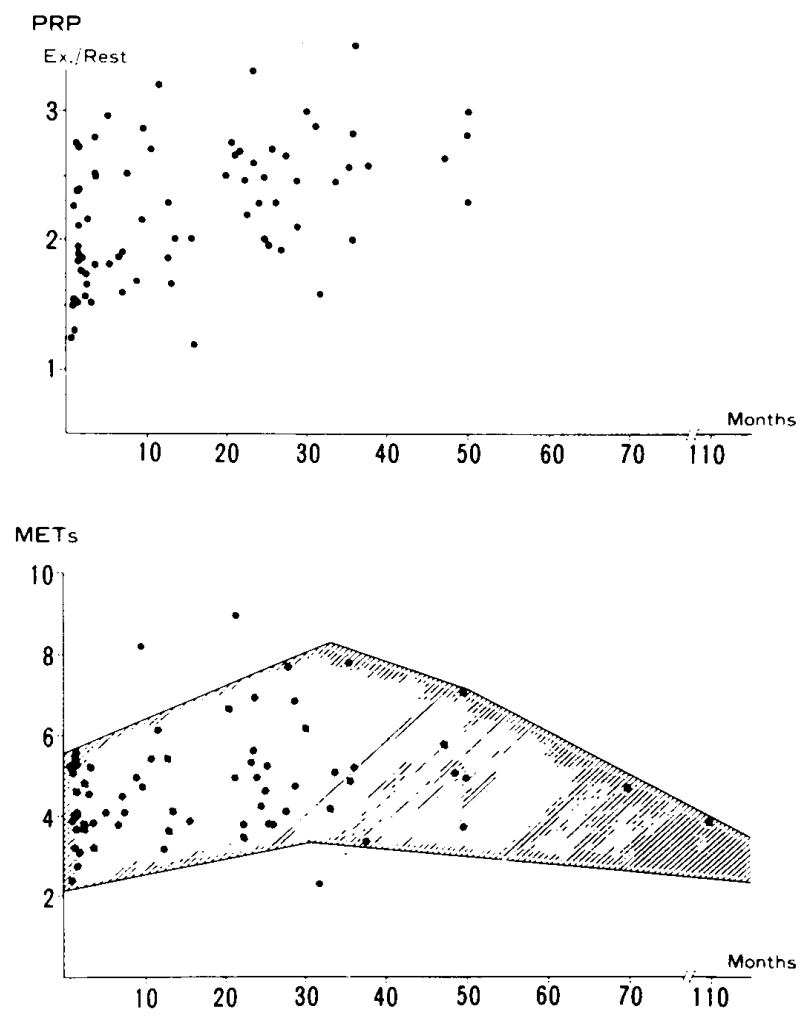

Fig.5. Time course of the PRP (upper panel), and the METs (lower panel) after the attack of myocardial infarction.

infarct size estimated from CPK levels correlated in patients during the 2.5-4.5 months after infarction. We measured only the peak CPK value, and thus, could not estimate the infarct size. This may be the reason why there was no correlation between CPK and the METs in the present study. However, cardiac function is one of the main determinants of physical capacity, which is affected by many other factors, such as peripheral circulation, oxygen extraction by the tissue, and enzymatic activity and mechanical efficacy of the muscles? Therefore, it is reasonable that there was no significant correlation between the METs and CPK, especially long after the acute attack.

There was a distinct correlation between age and the METs with a correlation coefficient of $r=-0.44(p<0.001)$. Excluding those patients who stopped exercise due to reaching the target heart rate, the correlation coefficient improved to -0.53 in patients within 6 months after their acute attack and -0.71 in patients after 6 months. Therefore, age is the most important determinant of physical capacity, especially when a long period has elapsed since the attack of acute myocaridal infarction. The slope of the correlation between age and the METs was -0.1 
(Fig. 3), being the same as that in normal subjects $!^{10}$ Assuming $1 \mathrm{MET}=3.5 \mathrm{ml} \mathrm{O} / \mathrm{kg} / \mathrm{min}$ and mean body weight $=70 \mathrm{~kg}$, the effect of age on an individual's physical capacity was the same in normal subjects as in patients with myocardial infarction 10

The PRP also showed a significant correlation with age in all cases $(\mathrm{r}=-0.41, \mathrm{p}<0.001)$, but there was no correlation between them in cases who were evaluated within 6 months after the acute attack. This suggests that in the early recovery phase, cardiac function is more markedly affected by the severity of infarction than by age, but afterwards the effect of age becomes more apparant. Fifty-six percent of the exercise tests were stopped because the age-predicted target heart rate was reached. This phenomenon may have affected the relationship between age and the PRP, but the same results were seen when the patients who reached their target heart rate were excluded.

Patients are now usually allowed to walk earlier than previously permitted in order to reduce cardiovascular deterioration after prolonged bed rest. This approach has been demonstrated to be safe in patients without complications!1,12 The PRP and the METs had no correlations with the duration of bed rest in this study. One of the reasons why our early ambulatory patients did not always have a better physical capacity is the criterion used before they were allowed to walk. The time interval between the acute attack and the exercise test varied from one month to 9 years, and therefore, the criterion was changed during the study period. However, we can speculate that without the continuation of physical exercise, the beneficial effects of early ambulation may be lost during a long-time period after the acute attack. As a matter of fact, patients who began to walk within 2 weeks had a higher physical capacity for 6 months after the attack.

Wohl et al ${ }^{3}$ followed the functional capacity of patients with myocardial infarction for some time by repeated exercise tests. They showed an increase of their capacity between the $3 \mathrm{rd}$ and the 6 th week, and between the $3 \mathrm{rd}$ and the 6 th month after the attack without any formal rehabilitation, but they did not follow up beyond 6 months. Uchijima et al ${ }^{13}$ monitored the physical capacity in myocardial infarction, and concluded that a remarkable improvement occurred within 6 months, and a gradual improvement continued up to 2-3 years after the attack. Our data showed that the METs were less than 5 in many cases during the early recovery phase, and that they increased 2-3 years after the attack (Fig. 5). The previous report from our laboratory ${ }^{14}$ also showed a physical capacity which improved during the $2-3$ years after the onset of myocardial infarction.

As shown in Fig. 5, the PRP varied over a wide range. The METs showed improvement while the PRP remained unchanged. This indicates that the improvement of physical capacity is mainly due to an increased efficacy of exercise and not due to an improvement of cardiac function.

\section{REFERENCES}

1. SALTIN B, BLOMQVIST CG, MITCHELL JH, JOHNSON RL Jr, WILDENTHAL K, CHAPMAN CB: Response to exercise after bed rest and after training. Circulation 38 (Suppl VII): 1, 1968

2. FROELICHER VF: Exercise and prevention of coronary atherosclerotic heart disease. In Exercise and Heart, ed by WENGER NK, Davis, Philadelphia, 1978, p 13

3. WOHL AJ, LEWIS HR, CAMPBELL W, KARLSSON E, WILLERSON JT, MULLINS CB, BLOMQVIST CG: Cardiovascular function during early recovery from acute myocardial infarction. Circulation 56: 931, 1977

4. PEEL AAF, SEMPLE T, WANG I, LANCASTER WM, DALL JLG: A coronary prognostic index for grading the severity of infarction. Br Heart $J 24$ : 745,1962

5. KITAMURA K, JORGENSEN CR, GOBEL FL, TAYLOR HL, WANG Y: Hemodynamic correlates of myocardial oxygen consumption during upright exercise. J Appl Physiol 32: 516, 1972

6. GOBEL FL, NORDSTROM LA, NELSON RR, JORGENSEN CR, WANG Y: The rate-pressure product as an index of myocardial oxygen consumption during exercise in patients with angina pectoris. Circulation 57: 549, 1978

7. CLAUSEN JP: Circulatory adjustments to dynamic exercise and effect of physical training in normal subjects and in patients with coronary artery disease. Prog Cardiovasc Dis 18: 459, 1976

8. GRANDE P, PEDERSEN A: Myocardial infarct size and cardiac performance at exercise soon after myocardial infarction. Br Heart J 47: 44, 1982

9. CARTER CL, AMUNDSEN LR: Infarct size and exercise capacity after myocardial infarction. $J$ Appl Physiol: Respirat Environ Exercise Physiol 42: 782, 1977

10. GERSTENBLITH G, LAKATTA EG, WEISFELDT ML: Age changes in myocardial function and exercise response. Prog Cardiovasc Dis 19: 1, 1976

11. BLOCH A, MAEDER J-P, HAISSLY J-C, FELIX J, BLACKBURN H: Early mobilization after myocardial infarction: A controlled study. $A m J$ Cardiol 34: 152, 1974

12. WEST RR, HENDERSON AH: Randomised multicentre trial of early mobilisation after uncomplicated myocardial infarction. $B r$ Heart $J$ 42: 
381,1979

13. UCHIJIMA H, SHIMMURA Y, MARUOKA T, HAYASHI M, HASEGAWA T, FUJITA Y: Follow-up study on cardiac function by exercise testing in patients with myocardial infarction after discharge. Jap J Rehabilit Med 18: 37, 1981 (in Japanese with English abstract)

14. TAKEUCHI M: Rehabilitation of the patients with myocardial infarction. Jpn Circ J 31: 1594, 1967 